\title{
Ontology Mining versus Ontology Speculation
}

\author{
Chris Partridge \\ BORO Solutions Limited, London, U.K. \\ Brunel University, Uxbridge, U.K. \\ partridgec@borogroup.co.uk
}

\begin{abstract}
When we embed the building of an ontology into an information system development or maintenance process, then the question arises as to how one should construct the content of the ontology. One of the choices is whether the construction process should focus on the mining of the ontology from existing resources or should be the result of speculation ("starting with a blank sheet of paper'). I present some arguments for choosing mining over speculation and then look at the implications this has for legacy modernisation.
\end{abstract}

